coloproctology $2020 \cdot 42: 6-13$

https://doi.org/10.1007/s00053-019-00418-8

Online publiziert: 26. November 2019

(c) Springer Medizin Verlag $\mathrm{GmbH}$, ein Teil von Springer Nature 2019

\author{
S. Petersen ${ }^{1} \cdot$ P. Holch ${ }^{2} \cdot$ J. Jongen ${ }^{3}$ \\ 'Allgemein- und Viszeralchirurgie, Asklepiosklinik Altona, Hamburg, Deutschland \\ ${ }^{2}$ Enddarmzentrum (EDZ) Eppendorf, Hamburg, Deutschland \\ ${ }^{3}$ Proktologische Praxis Kiel, Kiel, Deutschland
}

\title{
Leitlinien zur Behandlung des Hämorrhoidalleidens
}

\section{Ein Vergleich}

In den letzten Jahrzehnten haben Leitlinien (Guidelines, GL) eine wesentliche Bedeutung in der Behandlung gewonnen. Dabei sollten GL systematisch entwickelte Aussagen sein mit dem Ziel, eine angemessene und gesundheitsbezogene Versorgung spezifischer klinischer Situationen zu erreichen. Leitlinien dienen auch der Transparenz klinischer Entscheidungen und bringen somit Sicherheit für Patienten, aber auch für den Behandler. Dennoch stellen sie keine rechtlich verbindliche Anordnung dar, sondern sind lediglich eine Entscheidungshilfe, die an den individuellen Fall angepasst werden muss [11]. Bei gerichtlichen Streitigkeiten kann eine schwerwiegende Abweichung von der Leitlinie ohne spezielle Begründung beim individuellen Patienten durchaus problematisch werden.

Angesichts der extrem hohen Anzahl betroffener Patienten und Behandlungsmaßnahmen des Hämorrhoidalleidens - immerhin werden in Deutschland jährlich rechnerisch über $3 \mathrm{Mio}$. Behandlungsmaßnahmen durchgeführt haben Empfehlungen zur Therapie dieses Krankheitsbilds eine enorme Bedeutung $[14,15]$. In den letzten Jahren versuchten einzelne Verbände und Gesellschaften, Leitlinien zum Hämorrhoidalleiden zu erstellen [2, 6]. Bei der Vielzahl von Behandlungsoptionen und der Problematik der unsicheren Klassifikation war hier eine Zusammenfassung der Daten und die Ermittlung von LeitlinienEmpfehlungen überfällig. Nahezu zeitgleich sind in den letzten eineinhalb Jahren 3 Leitlinien zu Hämorrhoiden publiziert worden. Neben der GL der
Amerikanischen Vereinigung der Kolorektalchirurgen (ASCRS) entstand eine GL der European Society of Coloproctology (ESCP) und schließlich eine deutsche GL unter der wesentlichen $\mathrm{Fe}$ derführung der Deutschen Gesellschaft für Koloproktologie [7, 17, 23].

Ziel dieser Übersicht ist es, die 3 Leitlinien hinsichtlich ihrer Recherche-Technik, Aufarbeitung der Daten sowie Empfehlung zur stadienadaptierten Behandlung zu vergleichen.

Es kann nicht genug betont werden, dass es das große Verdienst der Autoren der GLs ist, aus der schier unübersichtlichen Flut der Daten Leitlinien entwickelt zu haben, die eine bessere Transparenz über die Therapieoptionen vermitteln und Handlungsempfehlungen aussprechen.

\section{Methoden}

Zunächst wurden die Methoden der Datengewinnung verglichen. Im nächsten Schritt erfolgte der Vergleich mit den Methoden, die in einzelnen Studien analysiert wurden.

\section{》) Die Qualität einer Leitlinie zeigt sich in ihrer internen sowie externen Validität}

In der weiteren Analyse werden die GLEmpfehlungen im Detail verglichen und eine Wertung abgegeben. Hierbei wurde insbesondere das für die Einstufung der Evidenz bzw. der Empfehlung verwendete Vorgehen untersucht.
Die Qualität einer Leitlinie zeigt sich im Vertrauen darauf, dass die evtl. vorhandenen systematischen Fehler der Leitlinienentwicklung angemessen angesprochen werden und die Empfehlungen sowohl interne als auch externe Validität besitzen. Dies bedeutet auch die Berücksichtigung von Nutzen sowie Limitationen der Empfehlungen. Deshalb umfasst die Bewertung Beurteilungen sowohl der zur Leitlinienentwicklung herangezogenen Methoden als auch des Inhalts der endgültigen Empfehlungen und der Faktoren, die mit der Realisierung der Empfehlungen verbunden sind. Zur Bewertung der Leitlinienqualität und -anwendbarkeit wurde in diesem Fall das AGREE-Instrument (Appraisal of Guidelines for Research \& Evaluation) herangezogen. Dieses Instrument wurde von einem internationalen Konsortium erarbeitet [3, 5]. Die Leitlinien wurden unabhängig voneinander von 3 Autoren durchgearbeitet.

\section{Ergebnisse}

\section{Vergleich der Methoden}

\section{ASCRS}

Die Literaturrecherche der amerikanischen GL stammt aus dem Jahr 2017. Basis der Behandlungsleitlinie war der Konsens eines 4-köpfigen Konsensusausschusses, bestehend aus einem Vorsitzenden, einem stellvertretenden Vorsitzenden sowie 2 beauftragten Gutachtern. Die Abschlussnote der Empfehlung und das Evidenzniveau für jede Aussage wurden anhand des GRADE-Bewertungssystems 
abgegeben. Die Mitglieder des ASCRS Clinical Practice Guidelines Committee erarbeiteten die Leitlinien bis zur endgültigen Veröffentlichung. Insgesamt erfolgte keine eng an den Daten orientierte Analyse. Die ASCRS-GL wurde mit keiner anderen amerikanischen Fachgesellschaft abgestimmt. Die Empfehlungen mit Evidenzgrad werden für einzelne Aspekte der Hämorrhoidalbehandlung abgegeben (s. unten).

\section{ESCP}

Die GL der europäischen Koloproktologie-Vereinigung wurden im Frühjahr 2019 fertiggestellt. Sie wurden von 9 europäischen Fachärzten, die mit der Behandlung von Hämorrhoiden befasst sind, erarbeitet. Am Anfang der Arbeit wurden klinisch wichtige Teilaspekte der Diagnostik und Behandlung in Frageform (sog. Schlüsselfragen) bearbeitet. Anhand dieser Schlüsselfragen wurde die Literaturrecherche im August 2017 durchgeführt. Die Auswertung der Daten erfolgte mit dem Cochrane Database of
Systematic Reviews System. Dafür wurden vergleichende, randomisierte und nichtrandomisierte Studien ausgewählt.

\section{DGK}

Für die deutsche GL wurde die Literatur mit dem Stichtag 01.07.2018 gesucht. Aus 6147 Publikationen wurden schließlich 1433 Literaturstellen extrahiert und bezüglich definierter Fragestellungen durchsucht. Dabei ist erwähnenswert, dass nicht nur vergleichende Analysen randomisierter oder nichtrandomisierte Art einbezogen wurden, sondern das gesamte Spektrum publizierter Daten von Fallberichten und Kurzmitteilungen bis hin zu Übersichtsarbeiten und systematischen Reviews einbezogen wurde. Diese Daten wurden von 21 Experten aus Österreich, der Schweiz und Deutschland ausgewertet. Die Darstellung der operativen Ergebnisse erfolgte in Evidenztabellen. Das Evidenzlevel einzelner Empfehlungen wurde in Anlehnung an die Empfehlungen des Centre for EvidenceBased Medicine angegeben.

\section{Methoden der GL}

Ein wichtiger Aspekt, insbesondere der ESCP war, dass in dieser GL Schlüsselfragen zu wichtigen Teilaspekten der Hämorrhoidalbehandlung gestellt wurden. In der amerikanischen GL wurden hingegen keine Schlüsselfragen genannt. In der deutschen GL steht zu den Schlüsselfragen geschrieben: Ziel der GL war die Behandlung folgender Themen, eine Reihenfolge zur Wichtigkeit der Themen/ Beschwerden/Ergebnisse wird nicht beschrieben. In der europäischen GL werden explizit die Schlüsselfragen benannt, an denen die Literatur durchgesucht und beurteilt und auch die Empfehlungen ausgesprochen wurden. So wurden als wichtig oder kritisch folgende Faktoren genannt: Restsymptomatik, Patientenzufriedenheit und Rezidive (Platz 1 bis 3). Nicht wichtig bzw. unkritisch wurden die Faktoren Kosten, Dauer der Operation sowie Dauer des Krankenhausaufenthalts eingestuft (Platz 8-10).

Hier steht eine Anzeige. 


\section{Einstufung der Empfehlung}

ASCRS und ESCP verwenden das GRADE-System, obwohl sie verschiedene Papers zum Grade-System zitieren. In der deutschen GL wird das System des Oxford Centre of Evidence-Based Medicine angewendet [19].

\section{Einstufung der Literatur (Evidenzlevel)}

Bezüglich der Literaturrecherche zeigt sich, dass die ASCRS-GL von 2018 auf der Literatur der ASCRS-GL von 2011 aufbaut und die aktuelle Literatur ergänzt wurde. Wie viele Personen mit der Beurteilung der Publikationen, die bei der Literaturrecherche gefunden wurden, bzw. bei der Formulierung der Empfehlungen beteiligt waren, ist unbekannt. Es wird lediglich berichtet, dass bei Unstimmigkeiten bezüglich Evidenz oder Empfehlung ein Konsens herbeigeführt wurde durch den Vorsitzenden der Kommission, den Stellvertreter und 2 weitere Reviewer. Die Leitlinie und Empfehlungen wurden gemeinsam mit der Leitlinienkommission der ASCRS diskutiert und modifiziert. Die endgültige Version der GL wurde mit den Empfehlungen durch die Leitlinienkommission und den Vorstand der ASCRS abgesegnet. Patienten waren an der Entwicklung der GL nicht beteiligt.

In der europäischen GL wird sehr genau beschrieben, dass nach der Formulierung der Schlüsselfragen eine Literaturrecherche durchgeführt wurde [12]. Sämtliche in der Recherche gefundenen Publikationen wurden anschlieBend durch einen Leitlinienexperten hinsichtlich ihrer Stärken und Schwächen eingeschätzt. Wenn systematische Reviews oder randomisiert-kontrollierte Studien (RCT) vorlagen, wurden andere (Fall-)Studien nicht (mehr) berücksichtigt. Fehlten RCT, dann wurde nach anderen Studien gesucht. Studien vor 1992 wurden nicht berücksichtigt. Die Beurteilung der Literatur, das Schreiben der Texte und Empfehlungen lag in den Händen von 3 Personen. Dieser Text mit den provisorischen Empfehlungen wurde anschließend allen Mitgliedern der Kommission zur Kommentierung zur Verfügung gestellt. Anschließend wur- den in der Leitlinienkommission unter Zuhilfenahme der GRADE-Tabellen die Empfehlungen formuliert und diskutiert und, wenn nötig, modifiziert. Die Diskussionen und Modifikationen fanden zu mehreren Gelegenheiten (Berlin, Amsterdam und Nizza) statt. Aus verschiedenen Ländern wurden Patienten gebeten, die GL durchzulesen und ihre Meinung aus Patientensicht zu geben [13]. Nach Fertigstellung der Leitlinie und Empfehlungen wurde die GL von der ESCP auf der Webseite publiziert. Alle ESCP-Mitglieder konnten einen Monat lang ihre Kritik und Bemerkungen einreichen. Die Leitlinie ist jetzt auf der ESCP-Webseite abrufbar. Eine Kurzfassung zur Publikation in Colorectal Disease ist geplant.

In der deutschen GL werden 1433 Literaturstellen (von 1965 bis 2017) gelistet. In verschiedenen Evidenztabellen wurden für jedes Thema die Publikationen eingestuft (z.B. Kapitel „Stapler“):

1. Fallserien,

2. Publikationen zum technischen Vorgehen oder mit anderen Instrumenten,

3. randomisiert-kontrollierte Studien,

4. systematische Reviews und Metaanalysen,

5. Publikationen zu postoperativen Veränderungen.

Die verschiedenen Kapitel der deutschen GL wurden von verschiedenen Mitgliedern der GL-Kommission geschrieben. Da verschiedene Mitarbeiter die Tabellen, Texte und Empfehlungen verfasst haben, ist ein Bias nicht auszuschließen. Die fertiggestellte GL wurde dann den verschiedenen Gesellschaften zur Verfügung gestellt. Anschließend wurde sie mit den Empfehlungen auf einer Konsensuskonferenz diskutiert und entsprechend (wenn notwendig) von den Vertretern der verschiedenen teilnehmenden Gesellschaften modifiziert. Wegen fehlender Patientenorganisation zu Hämorrhoidalleiden waren Patienten nicht an der Entwicklung der deutschen GL beteiligt. Eine Kurzfassung zur Publikation in coloproctology ist geplant.

\section{Leserlichkeit}

Die ASCRS-GL umfasst 9 Seiten; es gibt keine Langfassung. Die Langfassung der ESCP- und DGK-GL hat 82 bzw. 182 Seiten, sodass man dort nicht einfach schnell reinschauen kann. Kurzfassungen beider Leitlinien sind geplant. In der ESCP-GL sind die Empfehlungen vor dem Text platziert, zudem werden sie noch einmal im Begleittext aufgeführt. In der deutschen GL sind sie im Text eingebettet, sodass man sich zunächst einmal durch das Dokument arbeiten muss, bevor man die Empfehlung zu einer Therapie findet.

\section{Literaturliste}

Die ASCRS-GL hat 64 Literaturstellen (davon 27 [42\%] Cochrane-Studien, Metaanalysen oder RCT). Die ESCPund DGK-GL verfügen über jeweils 146 bzw. 1433 Stellen (53 [36\%] bzw. 272 (20\%) Cochrane-Studien, Metaanalysen oder RCT).

\section{Appraisal of Guidelines for Research and Evalution (AGREE II)}

Das AGREE-Instrument ermöglicht die Beurteilung der methodischen Genauigkeit und Transparenz der Leitlinienentwicklung (AGREE Collaboration. Development and validation of an international appraisal instrument for assessing the quality of clinical practice guidelines: the AGREE project [5]). Die Autoren haben die o.g. GL nach den AGREE-Kriterien beurteilt ( $\bullet$ Tab. 1). Es wird auf die Interessenkonflikte hingewiesen (Jos Kleijnen sowie Johannes Jongen waren an der Erstellung der ESCP-GL beteiligt, Johannes Jongen ebenfalls an der DGK-GL).

\section{Empfehlungen der GL im Detail}

Im Folgenden werden die Therapieempfehlungen der einzelnen GL detailliert dargestellt, eine Übersicht findet sich hierzu auch in • Tab. 2.

\section{Lokaltherapie}

Ballaststoffreiche Ernährung, ausreichende Flüssigkeitszufuhr, Beratung zum Stuhlgangverhalten werden in den 
3 GL empfohlen (ASCRS: starke Empfehlung, mit moderatem Evidenzlevel; ESCP: Expertenmeinung; DGK: B [1a LoV] bzw. 0 [2b LoE]).

Die Verwendung von Flavonoiden wird von der ASCRS und ESCP schwach empfohlen, mit niedrigem Evidenzlevel; in der Deutschen GL werden sie mit 0 (kann) und reduziertem 1AEvidenzlevel empfohlen, obwohl sie in Deutschland nicht zugelassen sind.

\section{Konservative Therapiemodalitäten}

In allen GL wird die Gummibandligatur (GBL) für Hämorrhoiden $1-3^{\circ}$ empfohlen. Die Sklerosierungstherapie mit Phenol oder einer Sulfatlösung wird bei Hämorrhoiden $1-3^{\circ}$ (ASCRS), Polidocanol oder Aluminiumsulfat (ESCP) bei Hämorrhoiden $1-2^{\circ}$ oder nur Polidocanol bei Hämorrhoiden $1-2^{\circ}$ (DGK) empfohlen. Die Infrarottherapie (IRC) wird von der ASCRS bei Hämorrhoiden 1-2 ${ }^{\circ}$ und von der ESCP bei blutenden Hämorrhoiden $1^{\circ}$ empfohlen. In der Deutschen GL kann sie bei blutenden niedriggradigen Hämorrhoiden eingesetzt werden.

\section{Operative Therapiemodalitäten}

In allen 3 Leitlinien besteht Konsensus, dass Hämorrhoiden 3. und 4. Grades eine Operationsindikation darstellen.

Die geschlossene Hämorrhoidektomie (nach Ferguson) wird im Vergleich zu den offenen Verfahren bei der ASCRS (weniger Schmerzen) und der ESCP (weniger Schmerzen und Blutungen) favorisiert. In der Deutschen GL werden die Verfahren nach Milligan-Morgan, Ferguson und Parks als gleichwertig beschrieben.

Alle GL sehen die Verwendung von bipolaren Scheren bzw. Ultraschallscheren bei der Exzisionshämorrhoidektomie als möglicherweise vorteilhaft an.

Das Staplerverfahren wird in der ESCP-GL für Hämorrhoiden $2-3^{\circ}$, in der DGK-GL für Hämorrhoiden $3^{\circ}$ empfohlen. Hämorrhoiden $4^{\circ}$ sind in der DGK-GL keine Indikation für ein Stapler-Verfahren. In der ESCP-GL wäre sie ein Alternative, wenn keine Hämorrhoidektomie möglich ist. In allen GL wird auf die erhöhte Rezidivrate und

coloproctology 2020 - 42:6-13 https://doi.org/10.1007/s00053-019-00418-8

(c) Springer Medizin Verlag GmbH, ein Teil von Springer Nature 2019

\section{S. Petersen $\cdot$ P. Holch $\cdot$ J. Jongen}

\section{Leitlinien zur Behandlung des Hämorrhoidalleidens. Ein Vergleich}

\section{Zusammenfassung}

Im letzten Jahr sind von den wichtigsten proktochirurgischen Fachgesellschaften Leitlinien (Guideline, GL) zur Behandlung des Hämorrhoidalleidens erarbeitet worden. Diese dienen der Transparenz für klinische Entscheidungen und bringen Sicherheit für Patienten, aber auch für den Behandler. Während bereits 2018 die Leitlinie der amerikanischen Kolorektalchirurgen (ASCRS) publiziert wurde, wurden die GL der Europäischen Vereinigung für Koloproktologie (ESCP) und die Leitlinie der Deutschen Gesellschaft für Koloproktologie (DGK) im Jahr 2019 veröffentlicht. Ziel dieser Übersicht ist es, die 3 Leitlinien hinsichtlich ihrer RechercheTechnik, Aufarbeitung der Daten sowie Empfehlung zur stadienadaptierten Behandlung zu vergleichen. Erwartungsgemäß zeigte sich, dass die verschiedenen Recherche-Techniken in den Leitlinien eine unterschiedliche
Anzahl von eingeschlossenen Publikationen ergab, wobei die DGK mit 1433 die höchste Anzahl von Arbeiten berücksichtigte. In Abhängigkeit der Konsensusfindung fallen die Behandlungsempfehlungen nicht gleichmäßig aus. Dennoch werden die bekannten konservativen und operativen Therapiemaßnahmen als anwendbar betrachtet, obgleich sich die Höhe des Evidenzniveaus und die Stärke der Empfehlung unterscheiden. Zusammenfassend ermöglichen es die neu erstellten Leitlinien, eine evidenzbasierte Behandlungsempfehlung abzugeben. Dabei wird das Evidenzniveau zwischen den GL auch auf Grundlage der unterschiedlichen Literaturdatenbasis abweichend eingeschätzt.

\section{Schlüsselwörter}

Hämorrhoiden · Chirurgie · Evidenz . Behandlungsempfehlung $\cdot$ Leitlinien

\section{Guidelines on treatment of hemorrhoids. A comparison}

\section{Abstract}

In the past year guidelines for the treatment of hemorrhoidal diseases have been developed by the main proctosurgery specialist societies. Guidelines serve to ensure transparency for clinical decisions and thus provide safety for patients as well as for the practitioner. While the guidelines of the American Society of Colon and Rectal Surgeons (ASCRS) were published in 2018, the guidelines of the European Society of Coloproctology (ESCP) and the guidelines of the German Society for Coloproctology (DGK) were published in 2019. The aim of this overview is to compare the three guidelines with respect to the research technique, processing of data and recommendations on stage-adapted treatment. As expected, the different research techniques in the guidelines took a different number of publications into account where the DGK took the highest number of publications into account with 1433 . The treatment recommendations are not uniform and depend on the consensus finding. Nevertheless, the known conservative and surgical treatment measures are considered applicable, although the level of evidence and the strength of the recommendations differ. The three guidelines published in 2018 and 2019 provide evidence-based guidance to surgeons who treat patients with hemorrhoids, although these guidelines differ in quality as evaluated according to the AGREE criteria.

\section{Keywords}

Hemorrhoids · Surgery · Evidence · Treatment recommendation - Guidelines das Auftreten von schwerwiegenden Komplikationen nach Stapler-Hämorrhoidopexie hingewiesen.

Die ASCRS-GL erwähnt die HALRAR-Methode, verweist aber auch auf die Hubble-Studie [4]. Die ESCP-GL sieht die Indikation zur HAL-RAR bei Hämorrhoiden $2-3^{\circ}$; allerdings stellt die Leitlinie den Sinn des Dopplers in Frage. Diese
Skepsis wird auch in der deutschen GL geteilt. Hämorrhoiden $3-4^{\circ}$ sollten nicht mit HAL-RAR therapiert werden, da die Methode mehr Rezidive hat als die konventionellen Operationsmethoden. 
Tab. 1 Einschätzung zur Qualität mittels des AGREE-Systems, die AGREE-Skala variiert von 1 („lowest possible quality") bis 7 ("highest possible quality")

\begin{tabular}{l|l|l|l|}
\hline Leitlinie & $\begin{array}{l}\text { Jos } \\
\text { Kleijnen }\end{array}$ & $\begin{array}{l}\text { Sven } \\
\text { Petersen }\end{array}$ & $\begin{array}{l}\text { Johannes } \\
\text { Jongen }\end{array}$ \\
\hline ASCRS & 2 & 2 & $2-3$ \\
\hline ESCP & 6 & 6 & 6 \\
\hline DGK & 3 & 5 & 4
\end{tabular}

\section{Antikoagulanzien bzw. Thrombo-} zytenaggregationshemmer

Die ASCRS erwähnt eine japanische Studie, die nach Sklerotherapie bei Patienten mit und ohne Blutverdünnern keinen Unterschied hinsichtlich Blutungen feststellte [25]. Die ESCP-GL nimmt bezüglich der Frage der Antikoagulation keine Stellung und verweist auf die verschiedenen nationalen Leitlinien zur Therapie mit Antikoagulanzien und Thrombozytenaggregationshemmern. Die DGKGL erklärt (als Expertenmeinung), dass ASS allein keine Kontraindikation für irgendeine Hämorrhoidaltherapie darstellt. Nimmt der Patient zusätzlich Clopidogrel, Phenprocoumon usw. ein, kann lediglich eine Sklerosierungstherapie durchgeführt werden, oder der Patient muss umgestellt werden.

\section{Chronisch-entzündliche Darmerkrankungen, Radiatio, Immunsuppression}

In der ASCRS-GL werden diese Konstellationen nicht erwähnt. Die ESCPGL empfiehlt, bei Patienten mit chronisch-entzündlichen Darmerkrankungen (CED) eine Hämorrhoidalbehandlung lediglich in der Remissionsphase durchzuführen. Bei zuvor bestrahlten Patienten sollte man ebenfalls zurückhaltend sein. Bei immunkompromittierten (HIV-positiven) Patienten sollte eine Therapie nur durchgeführt werden, wenn diese „fit" sind. In der deutschen GL werden CED- und HIV-Patienten zwar erwähnt, jedoch wird keine Empfehlung ausgesprochen.
Patientinnen mit postpartalen Hämorrhoidalbeschwerden oder während Schwangerschaft

In der ASCRS-GL werden diese Patientinnen nicht erwähnt, in den anderen GL wird stets zu einem konservativen Vorgehen geraten.

\section{Thrombosierte Hämorrhoiden/ Analvenenthrombose}

In der ASCRS-GL wird ein operatives Vorgehen schwach empfohlen bei niedrigem Evidenzlevel (2C). Die ESCP-GL empfiehlt ein konservatives Vorgehen (Expertenmeinung), eventuell können Phlebotonika eingesetzt werden (niedriges EL). In auserwählten Fällen kann operativ vorgegangen werden (sehr niedriges EL). In der deutschen GL wird bei thrombosierten Befunden bzw. Analvenenthrombosen zu einem konservativen Vorgehen geraten.

\section{Diskussion}

Hämorrhoiden sind ein Gesundheitsproblem, das viele Patienten weltweit betrifft. In Deutschland werden viele Patienten ambulant therapiert, etwa 50.000 werden hierzulande wegen eines Hämorrhoidalleidens operiert. In den letzten Jahren sind verschiedene Leitlinien publiziert worden: italienische, französische, amerikanische, niederländische, deutsche sowie europäische. Da die meisten Leitlinien den Anspruch haben, evidenzbasiert zu sein, wäre anzunehmen, dass sie im Prinzip eigentlich alle identisch sein müssten.

Während die ESCP-GL die statische Analyse des Cochrane-Systems nutzt, um Daten zu analysieren und Aussagen zu formulieren, nutzen die amerikanischen und die deutschen GL die Konsensusfindung als wesentliche Methode. Beide Analysetechniken haben Vor- und Nachteile und die Diskussion um die sicherste Aussage hält seit vielen Jahrzehnten an [18]. Der Vorteil einer Metaanalyse aus randomisierten bzw. vergleichenden, aber nichtrandomisierten Studien ist, dass die Daten aus gleichen Instituten stammen und die Fragestellung im Idealfall bereits vor der Behandlung fest- gelegt war. Auf der anderen Seite wurde in den letzten Jahren deutlich, dass beispielsweise randomisierte Studien zum Thema Behandlung des Hämorrhoidalleidens aus Universitätskliniken mit einem teilweise extrem geringen Caseload publiziert wurden. Es ist eine Binsenweisheit, dass die Analysen nur so gut sein können wie die Literatur, die ihnen zugrunde liegt. Dies gilt gleichermaßen für die GL der ASCRS, ESCP und DGK. Aber was ist eine randomisierte Studie wert, bei der nur ein Fall pro Monat pro Institut randomisiert wird? Ebenso bleibt die Frage, was eine Metaanalyse wert ist, die aus diesen Rohdaten errechnet wird? Bei näherer Analyse von Metaanalysen erhält man häufig den Eindruck, dass nur die Abstracts und nicht die Originalpublikationen analysiert wurden [16]. Es muss berücksichtigt werden, dass die Fall-Kontroll-Studien mit retrospektiver oder prospektiver Erfassung von Ergebnissen der Hämorrhoidenbehandlung die Wirklichkeit wahrscheinlich viel eher abbilden als randomisierte Studien. Die nichtvergleichenden Daten einzelner Institute mit tausenden von Behandlungen blieben sonst unberücksichtigt [20, 24]. Daher kann das Verdienst der DGKLeitlinie nicht hoch genug angerechnet werden, dass sich die Kollegen/Autoren die Mühe gemacht haben, auch Fall-Kontroll-Studien und Fallberichte in die Konsensusdiskussion aufzunehmen.

Neben der wissenschaftlichen Evidenz wird die Behandlungsempfehlung wesentlich durch das jeweilige Gesundheitssystem beeinflusst. In Deutschland werden die Hämorrhoidaltherapien meist ambulant im niedergelassenen Bereich durchgeführt. Die meisten Operationen wegen Hämorrhoidalleidens erfolgen jedoch nach wie vor unter stationären Bedingungen. Für eine Gummibandligatur gibt es beispielsweise keine OPS/DRG (Operations- und Prozedurenschlüssel/ Diagnosen-bezogene Fallgruppen)! In den verschiedenen europäischen Ländern bestehen andere Gesundheitssysteme und Zuständigkeiten: In Frankreich führen Gastroenterologen auch manche proktologischen Operationen (!) durch. In Italien braucht der Patient eine unter stationären Bedingungen durchgeführte Therapie nicht zu bezahlen. In Dänemark 


\begin{tabular}{|c|c|c|c|}
\hline & ASCRS 2018 & ESCP 2019 & DGK 2019 \\
\hline Literatur & 1996-2017 & 1992-2017 & 1965-2017 \\
\hline Anzahl Literaturstellen & 64 & 146 & 1433 \\
\hline $\begin{array}{l}\text { Cochrane/systemisches } \\
\text { Review/randomisiert- } \\
\text { kontrollierte Studien (\%) }\end{array}$ & $27(42 \%)$ & $53(36 \%)$ & $272(20 \%)$ \\
\hline Anzahl Seiten & 9 & 93 (Langfassung) & 182 (Langfassung) \\
\hline Grading/EL & $\begin{array}{l}\text { GRADE } \\
{[9,22]}\end{array}$ & $\begin{array}{l}\text { GRADE } \\
\text { [19] } \\
\text { EL: hoch, moderat, niedrig und sehr } \\
\text { niedrig }\end{array}$ & $\begin{array}{l}\text { Centre for Evidence-Based Medicine, } \\
\text { Oxford, UK } \\
{[10]}\end{array}$ \\
\hline $\begin{array}{l}\text { Benutztes System für } \\
\text { Empfehlung (Evidenz) }\end{array}$ & GRADE & GRADE & $\begin{array}{l}\text { A: starke E (soll) } \\
\text { B: E (sollte) } \\
\text { O: E offen (kann/unklar) }\end{array}$ \\
\hline Einstufung & $\begin{array}{l}\text { 1A: starke E (EL hoch) } \\
1 \mathrm{~B} \text { : starke } \mathrm{E} \text { (EL moderat) } \\
\text { 1C: stark E (EL niedrig oder sehr nied- } \\
\text { rig) } \\
\text { 2A: schwache E (EL hoch) } \\
\text { 2B: schwache E (EL moderat) } \\
\text { 2C: schwache E (EL niedrig oder sehr } \\
\text { niedrig) }\end{array}$ & $\begin{array}{l}\text { Must/must not: EL ++++ } \\
\text { Should/should not: } \\
\text { EL: }+++ \\
\text { Could/could not: } \\
\text { EL: }++ \\
\text { Can be considered: } \\
\text { EL: }+\end{array}$ & $\begin{array}{l}\text { Soll: } A(1 \mathrm{a}, 1 \mathrm{~b}, 1 \mathrm{c}) \\
\text { Sollte: } \mathrm{B}(2 \mathrm{a}, 2 \mathrm{~b}, 2 \mathrm{c}) \\
\text { Kann: } 0(3 \mathrm{a}, 3 \mathrm{~b}, 4,5) \\
\text { Zusätzlich Konsensstärke angegeben }\end{array}$ \\
\hline Anzahl der Mitglieder & 4 (Chirurgen) & $\begin{array}{l}9 \text { (Chirurgen, Gastroenterologe, Allge- } \\
\text { meinmediziner) }\end{array}$ & $\begin{array}{l}20 \text { (Chirurgen, Dermatologen, Gastro- } \\
\text { enterologen, Allgemeinmediziner) }\end{array}$ \\
\hline $\begin{array}{l}\text { Andere Gesellschaften } \\
\text { involviert? }\end{array}$ & Nein & $\begin{array}{l}\text { Nein, nur Mandatsträger nationaler } \\
\text { Gesellschaften }\end{array}$ & Ja \\
\hline Häm. Einteilung & Goligher [8] & Goligher [8] & Goligher [8] \\
\hline Flavonoide & $2 C[1,21]$ & Low-level (++) & 0 (EL: $1 \mathrm{~A}$ minus) \\
\hline $\begin{array}{l}\text { Konservative Therapie } \\
\text { Sklerotherapie, IRC, RBL }\end{array}$ & $\begin{array}{l}1 \mathrm{~A}, \mathrm{RBL} \text { most effective } \\
\text { Häm. } 1 \text { und } 2^{\circ} \text {, selekt. Pat- mit Häm. } 3^{\circ}\end{array}$ & - & - \\
\hline Gummibandligatur (RBL) & Most effective & RBL Häm. 1-3 (++) & $\begin{array}{l}\text { Häm. 1-3: } 0 \text { (EL: 4) } \\
\text { Häm. 2: B (EL: 1b) } \\
\text { Häm. 2-3: B (EL: 1b) } \\
\text { Häm. 2-3: im Vergleich zu Op.: } 0 \text { (EL } \\
\text { 1a) }\end{array}$ \\
\hline Sklerotherapie & $\begin{array}{l}\text { Phenol oder sodium tetradecyl sulfate } \\
\text { Häm. } 1-3^{\circ}\end{array}$ & $\begin{array}{l}\text { Scl: Häm. 1-2 (+) } \\
\text { polidocanol or aluminium potassium } \\
\text { sulfate }\end{array}$ & $\begin{array}{l}\text { Es sollte nur Polidocanol benutzt } \\
\text { werden } \\
\text { Häm. 1-2: } 0 \text { (EL: 4) }\end{array}$ \\
\hline IRC & Häm 1-2 & IRC blutende Häm. 1 (+) & $\begin{array}{l}\text { Bei niedriggradigen Hämorrhoiden } \\
\text { mit Blutungen ( } 0 \mathrm{EL} \text { 1a minus) }\end{array}$ \\
\hline Operation & $\begin{array}{l}\text { External hemorrhoids or combined } \\
\text { internal and external hemorrhoids } \\
\text { with prolapse (grades III-IV) } \\
1 \mathrm{~A}\end{array}$ & $\begin{array}{l}\text { Häm. 3-4 } \\
\text { DGHAL bei Häm 2-3 } \\
\text { Hämorrhoidektomie/Stapler bei Häm. } \\
2-4^{\circ}, \text { SH weniger effektiv als Hämor- } \\
\text { rhoidektomie insbes. bei Häm. } 4^{\circ}\end{array}$ & $\begin{array}{l}\text { Häm. } 3-4^{\circ} \text { oder bei Versagen der } \\
\text { konservativen Therapie } \\
\text { Segm. Verfahren (Milligan-Morgan } \\
\text { Ferguson, Parks) gleichwertig (A EL 1a) }\end{array}$ \\
\hline $\begin{array}{l}\text { Milligan-Morgan/ } \\
\text { Ferguson }\end{array}$ & $\begin{array}{l}\text { Ferguson weniger Schmerzen als } \\
\text { Milligan-Morgan } \\
\text { Bipolare bzw. Ultraschallschere evtl. } \\
\text { besser bzgl. Arbeitsunfähigkeit und } \\
\text { postoperativer Schmerzen, Kosten } \\
\text { sind höher }\end{array}$ & $\begin{array}{l}\text { Ferguson weniger Schmerzen und } \\
\text { Blutungen als Milligan-Morgan, } \\
\text { Häm. 2-3: EL: ++ } \\
\text { Häm. } 4^{\circ} \text { : EL: ++ }\end{array}$ & $\begin{array}{l}\text { Bipolare bzw. Ultraschallschere bei } \\
\text { Milligan-Morgan/Ferguson (A E 1a) }\end{array}$ \\
\hline Stapler & $\begin{array}{l}\text { Ethos-Studie erwähnt [4] } \\
\text { Mehr Rezidivprolaps, mehr Zusatzope- } \\
\text { rationen } \\
\text { Mehr und schwerwiegendere Kompli- } \\
\text { kationen! }\end{array}$ & $\begin{array}{l}\text { Häm. } 2-3^{\circ} \\
\text { EL: }++ \\
\text { Ethos-Studie aufgenommen }\end{array}$ & $\begin{array}{l}\text { Häm. } 3^{\circ} \text { zirkulär (A EL 1b) } \\
\text { Häm. } 4^{\circ} \text { keine Indikation für Stapler (B } \\
\text { 1b) } \\
\text { Mehr Rezidive im Vergleich zu konven- } \\
\text { tionellen Verfahren (EL 1a) }\end{array}$ \\
\hline
\end{tabular}




\begin{tabular}{|c|c|c|c|}
\hline & ASCRS 2018 & ESCP 2019 & DGK 2019 \\
\hline $\begin{array}{l}\text { Hämorrhoidenarterien- } \\
\text { ligatur-Rektoanal-Repair } \\
\text { (HAL-RAR) }\end{array}$ & Hubble-Studie erwähnt [4] & $\begin{array}{l}\text { Hubble-Studie erwähnt [4], } \\
\text { HAL-RAR ++ } \\
\text { Nur RAR + }\end{array}$ & $\begin{array}{l}\text { Bei RAR kann auf den Doppler verzich- } \\
\text { tet werden (B EL } 2 \text { b) } \\
\text { Nicht bei Häm. 3-4, weil mehr Rezi- } \\
\text { dive im Vergleich zu konventionellen } \\
\text { Methoden (EL 1a) }\end{array}$ \\
\hline Postoperative Schmerzen & Multimodality pain regimen $1 \mathrm{~B}$ & - & $\begin{array}{l}\text { Glyceroltrinitrat oder Kalziumantago- } \\
\text { nisten lokal (0 EL 1a) } \\
\text { Metronidazol sollte nicht routinemä- } \\
\text { Big verabreicht werden (B EL 1a) } \\
\text { Pudendusblock bei Allgemeinanäs- } \\
\text { thesie (B EL 1a) } \\
\text { Quellmittel (0, EL 2b) }\end{array}$ \\
\hline Antikoagulation & Nur bei Sklerotherapie erwähnt & $\begin{array}{l}\text { Änderung/Aufhören nach nationalen } \\
\mathrm{GL}\end{array}$ & $\begin{array}{l}\text { Ja } \\
\text { ASS: alle Methoden erlaubt } \\
\text { ASS, Clopidogrel, Phenprocoumon, } \\
\text { NOAK } \rightarrow \text { nur Sklerotherapie, sonst } \\
\text { Umstellung! }\end{array}$ \\
\hline $\begin{array}{l}\text { Thrombosierte } \\
\text { Hämorrhoiden/ } \\
\text { Analvenenthrombose }\end{array}$ & Op.: $2 \mathrm{C}$ & $\begin{array}{l}\text { Konservativ: Expertenmeinung: Flavo- } \\
\text { noid: }++, \text { Op.: }+\end{array}$ & $\begin{array}{l}\text { Primär konservativ (Expertenmei- } \\
\text { nung) }\end{array}$ \\
\hline CED & Nicht erwähnt & Höchstens in Remission & Keine Empfehlung \\
\hline Radiatio & Nicht erwähnt & Erwähnt, eher kontraindiziert & - \\
\hline $\begin{array}{l}\text { Immunkompromittierte } \\
\text { Patienten }\end{array}$ & Nicht erwähnt & Wenn die Patienten "fit" sind & Keine Empfehlung \\
\hline Schwangere /postpartale & Nicht erwähnt & Konservativ & Konservativ (Expertenmeinung) \\
\hline Komplikationen & Werden diskutiert & Werden diskutiert & Werden diskutiert \\
\hline
\end{tabular}

kann es sein, dass ein Patient mit Hämorrhoiden, der stationär eingewiesen wird, eine Operation erhält, auch wenn womöglich eine Gummibandligatur eher sinnvoll gewesen wäre. In Großbritannien wird das gesamte Spektrum vielleicht differenziert durchgeführt, für notwendige/indizierte Operationen bestehen aber häufig Wartezeiten (Hubble-Studie: bis $\mathrm{zu} 9$ Monate für HAL-RAR; [4]). Da in allen Leitlinien keine scharfe Trennung zwischen den Indikationen zur Therapie gemacht wird, können die Leitlinien sowohl in Richtung konservative Therapie, aber auch in Richtung Operation ausgelegt werden.

\section{Fazit für die Praxis}

- Zusammenfassend ermöglichen es
die neu erstellten Leitlinien, eine
evidenzbasierte Behandlungsemp-
fehlung abzugeben.
- Das Evidenzniveau zwischen den
Guidelines (GL) wird auch auf Grund-
lage der unterschiedlichen Literatur-

datenbasis abweichend eingeschätzt.

\section{Korrespondenzadresse}

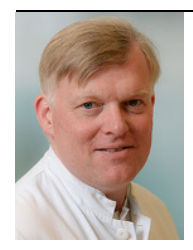

PD Dr. S. Petersen

Allgemein- und Viszeralchirurgie, Asklepiosklinik Altona

Paul-Ehrlich-Str. 1,

22763 Hamburg, Deutschland sv.petersen@asklepios.com

Danksagung. Die Autoren danken Herrn Prof. Jos Kleijnen, Maastricht University, für die Hilfestellung zur Bewertung mit dem AGREE-System.

\section{Einhaltung ethischer Richtlinien}

Interessenkonflikt. S. Petersen gibt an, dass folgende Interessenkonflikte bestehen: in den letzten 5 Jahren wurde Zuwendungen von den Firmen Johnson \& Johnson, Ethicon Endosurgery (Norderstedt, Deutschland), THD Deutschland (München, Deutschland) und DACH-Medical (Ostermiething, Österreich) angenommen, J. Jongen war an der Erstellung der ESCP- und deutsche Leitlinie Hämorrhoiden beteiligt; P. Holch gibt an, dass kein Interessenkonflikt besteht.
Für diesen Beitrag wurden von den Autoren keine Studien an Menschen oder Tieren durchgeführt. Für die aufgeführten Studien gelten die jeweils dort angegebenen ethischen Richtlinien.

\section{Literatur}

1. Alonso-Coello P, Zhou Q, Martinez-Zapata MJ et al (2006) Meta-analysis of flavonoids for the treatment of haemorrhoids. Br JSurg 93:909-920

2. Altomare DF, Roveran A, Pecorella Getal (2006) The treatment of hemorrhoids: guidelines of the Italian Society of Colorectal Surgery. Tech Coloproctol 10:181-186

3. Appraisal of Guidelines, Research, and Evaluation in Europe (AGREE) Collaborative Group (2000) Guidelinedevelopmentin Europe. An international comparison. Int J Technol Assess Health Care 16(4):1039-1049

4. Brown SR, Tiernan JP, Watson AJM et al (2016) Haemorrhoidal artery ligation versus rubber band ligation for the management of symptomatic second-degree and third-degree haemorrhoids (HubBLe): a multicentre, open-label, randomised controlled trial. Lancet 388:356-364

5. AGREE Collaboration (2003) Development and validation of an international appraisal instrument for assessing the quality of clinical practice guidelines: the AGREE project. Qual Saf Health Care 12:18-23

6. D'ugo S, Franceschilli L, Cadeddu F et al (2013) Medical and surgical treatment of haemorrhoids 
and anal fissure in Crohn's disease: a critical appraisal. BMC Gastroenterol 13:47

7. Davis BR, Lee-Kong SA, Migaly J et al (2018) The American society of colon and rectal surgeons clinical practice guidelines for the management of hemorrhoids. Dis Colon Rectum 61:284-292

8. Goligher JC, Duthie H, Nixon H (1975) Surgery of the anus, rectum and colon. Baillière Tindall, London

9. Guyatt G, Gutterman D, Baumann MH et al (2006) Grading strength of recommendations and quality of evidence in clinical guidelines: report from an american college of chest physicians task force. Chest 129:174-181

10. CEBM Webpräsenz. http://www.cebm.net. Zugegriffen: 2019

11. Cochrane Deutschland Webpräsenz. http://www. cochrane.de/.Zugegriffen: 2019

12. European Society of Coloproctology (ESCP) Processes and methods. https://www.escp.eu.com/ Guidelines/Processes-and-Methods. Zugegriffen: 2019

13. European Society of Coloproctology (ESCP) (2018) Feedback welcomed on new ESCP haemorrhoidal guidelines. https://www.escp.eu.com/
News/1797-Feedback-Welcomed-on-New-EscpHaemorrhoidal-Guidelines. Zugegriffen: 24. Dez. 2018

14. Johanson JF, Sonnenberg A (1990) The prevalence of hemorrhoids and chronic constipation. An epidemiologic study. Gastroenterology 98:380-386

15. Jongen J, Kahlke V (2019) Quality indicators in the treatment of hemorrhoids. Chirurg 90:264-269

16. Jongen J, Kahlke V, Petersen S (2015) Concerns regarding the selection of literature and extraction of data in published reviews and meta-analyses. Dis Colon Rectum 58:e444

17. Joos AK, Arnold R, Borschitz T et al (2019) S3Leitlinie - Hämorrhoidalleiden (AWMF-Registriernummer:081/007)

18. Mantel N, Haenszel W (1959) Statistical aspects of the analysis of data from retrospective studies of disease. J Natl Cancer Inst 22:719-748

19. Neumann I, Pantoja T, Penaloza B et al (2014) The GRADE system: a change in the way of assessing the quality of evidence and the strength of recommendations. Rev Med Chil 142:630-635

20. $\mathrm{Ng} \mathrm{KH}, \mathrm{HoKS}$, Ooi BS et al (2006) Experience of 3711 stapled haemorrhoidectomy operations. Br J Surg 93:226-230
21. Perera N, Liolitsa D, lype S et al (2012) Phlebotonics for haemorrhoids. Cochrane Database Syst Rev. https://doi.org/10.1002/14651858.CD004322. pub3

22. Schunemann HJ, Jaeschke R, Cook DJ et al (2006) An official ATS statement: grading the quality of evidence and strength of recommendations in ATS guidelines and recommendations. Am J Respir Crit Care Med 174:605-614

23. van Tol RR, Kuiper SZ, Watson AJM et al (2019) Guideline regarding treatment of haemorrhoids. https://www.escp.eu.com/guidelines\# haemorrhoids. Zugegriffen: 2019

24. Voigtsberger A, Popovicova L, Bauer G et al (2016) Stapled hemorrhoidopexy: functional results, recurrence rate, and prognostic factors in a single center analysis. Int J Colorectal Dis 31:35-39

25. Yano T, Nogaki T, Asano Metal (2013) Outcomes of case-matched injection sclerotherapy with a new agent for hemorrhoids in patients treated with or without blood thinners. Surg Today 43:854-858

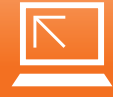

\section{Praxis-Finanzrechner für Ärzte Neues Tool auf SpringerMedizin.de}

Wie rentabel läuft meine Praxis? Wie stehe ich im Vergleich zu meinen Kollegen im Bundesland? Welche Anschaffungen kann ich mir leisten? Antworten auf diese betriebswirtschaftlichen Fragen liefert künftig ein Tool auf SpringerMedizin.de, indem es aktuelle Versorgungs- und Abrechnungsdaten sowie Analysedaten deutscher Gesundheitsunternehmen zu Grunde legt.

Mit den kostenfreien Praxis-Finanzrechnern können sich vor allem in der Praxis tätigen Ärzte, die sich oft auch unternehmerischen Herausforderungen stellen müssen, interaktiv mit den Themen Controlling und Benchmarking auseinandersetzen:

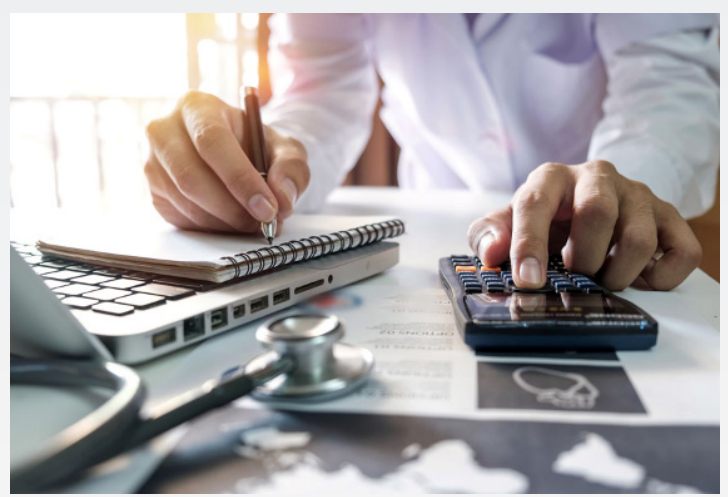

(c) mrmohock/stock.adobe.com
Mit dem Controlling-Assistenten behalten Sie die wichtigsten wirtschaftlichen Praxiskennzahlen immer im Auge. $>$ Mit dem Tool Benchmark können Sie Ihre Praxis mit anderen Praxen vergleichen.

$>$ Ihren Praxisstandort - ebenfalls ein wichtiger Faktor für den Erfolg - analysieren Sie mit Standortcheck aus verschiedenen Perspektiven

Mit dem Investitionsassistenten können sie sehen, ob und wann sich eine Geräteinvestition amortisiert und Sie bekommen auch Anhaltspunkte, welche Einnahmen Sie zu erwarten haben.

Die Praxis-Tools sind ein externes Angebot von Rebmann Research, Partner von SpringerMedizin.de. Sie finden die Praxis-Finanzrechner auf SpringerMedizin.de unter dem Navigationspunkt "Mehr"

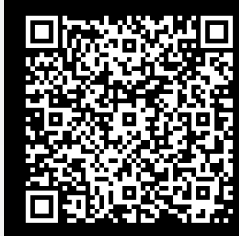

